\title{
Spontaneous nystagmus in dorsolateral medullary infarction indicates vestibular semicircular canal imbalance
}

\author{
H Rambold, C Helmchen
}

J Neurol Neurosurg Psychiatry 2005;76:88-94. doi: 10.1136/jnnp.2003.031690

See end of article for authors' affiliations

.....................

Correspondence to: Dr Holger Rambold, Department of Neurology, University of Luebeck, Ratzeburger Allee 160, D-23538 Luebeck, Germany; rambold h@ neuro.mu-luebeck.de

Received 4November 2003 In revised form 8 April 2004 Accepted 27 April 2004
Background: Spontaneous nystagmus caused by dorsolateral medullary infarction may be of vestibular origin.

Objectives: To test if imbalance of the central pathways of the semicircular canals contributes to spontaneous nystagmus in dorsolateral medullary syndrome.

Methods: We examined four patients with dorsolateral medullary syndrome and recorded spontaneous nystagmus binocularly at gaze straight ahead with the three-dimensional search coil technique. The median slow phase velocity of the nystagmus was analysed in the light and in the dark, and the normalised velocity axes were compared with the rotation axes as predicted from anatomical data of the semicircular canal.

Results: The slow phase rotation axes of all patients aligned best with the rotation axes resulting from stimulation of the contralesional posterior and horizontal semicircular canals. This alignment cannot be explained by pure otolith imbalance.

Conclusion: We propose that vestibular imbalance caused by an ipsilesional lesion of the central semicircular canal pathways of the horizontal and anterior semicircular canals largely accounts for spontaneous nystagmus in dorsolateral medullary syndrome.
$\mathrm{V}$ ertigo, ipsilesional lateropulsion, and ipsilesional ocular tilt reaction (OTR, skew deviation with hypotropia of the eye on the lesion side, ocular torsion, and head tilt to the side of the lesion) are major features of dorsolateral medullary infarction. ${ }^{12}$ These symptoms may be caused by imbalance of central otolith signals due to impairment of the caudal part of the vestibular nucleus complex..-5 There is some evidence that in addition to central otolith imbalance, imbalance of the central pathways of the semicircular canals also contributes to the vestibular signs in dorsolateral medullary infarction. ${ }^{67}$ In the early phase of dorsolateral medullary infarction, spontaneous nystagmus is often observed at gaze straight ahead with a horizontal, ${ }^{67}$ torsional, ${ }^{6}$ or mixed torsional, horizontal, and vertical direction component. ${ }^{6} 8$ The nystagmus normally beats to the intact side, ${ }^{268}$ but it may rarely also beat to the side of the infarction. ${ }^{279}$ Linear, exponential increasing and decreasing drifts are found during the slow phases. Nystagmus in these patients is often present only in the first days and rapidly declines over the following days. Its pathomechanism could be vestibular imbalance ${ }^{4}$ caused by a lesion of the vestibular nuclei or their commissural pathways in the dorsal medulla. However, analysis of the nystagmus with respect to the rotation axes of the semicircular canals has been missing so far. $^{6}{ }^{10}$ To analyse the contribution of the individual semicircular canals to spontaneous nystagmus we recorded binocular, three-dimensional (3-D) eye movements of four patients with dorsolateral medullary infarction under head restrained conditions and analysed the rotation axes of the slow phases. We compared the slow phase axes with those of the semicircular canals obtained from anatomical data. ${ }^{11}$

Preliminary data have been published in abstract form

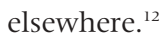

\section{METHODS}

Patient \# 1

A 66 year old man presented with left dorsolateral medullary syndrome. On admission he had left Horner's syndrome, sensory loss of pain and temperature on the left side of his face, right trunk, and limbs, and hemiataxia of the left side. He also showed a falling tendency to the left. Oculomotor examination demonstrated conjugate nystagmus in gaze straight ahead with a clockwise (rightward) torsional (contralesionally beating fast phases; from the subject's point of view) and a rightward horizontal component. The torsional component was more pronounced in the right eye; it increased in amplitude but decreased in frequency in the dark. There was a small vertical component in the upward direction. The patient had leftward lateropulsion of saccades (hypermetria), saccadic smooth pursuit to the right, skew deviation with hypotropia of the left eye, and a head tilt to the left. Magnetic resonance imaging (MRI) revealed a left dorsolateral medullary infarction (fig 1). Eye movement recordings were performed 1 day after symptom onset.

\section{Patient \#2}

A 64 year old woman presented with left dorsolateral medullary syndrome. Left Horner's syndrome, sensory loss of pain and temperature on the left side of the face and her right trunk and limbs, and hemiataxia of her left side were present. She also showed a falling tendency to the left. Oculomotor examination detected nystagmus with clockwise (contralesionally beating, rightward) torsional, rightward horizontal, and upward vertical fast phase components that increased in amplitude in the dark. The torsional nystagmus had a larger torsional component in the right eye. The patient had leftward lateropulsion of saccades (hypermetria), saccadic smooth pursuit eye movements to the right, skew deviation with hypotropia of the left eye, and a head tilt to the left. MRI revealed a left dorsolateral medullary infarction (fig 1). Eye movement recordings were performed 1 day after symptom onset.

Abbreviations: MRI, magnetic resonance imaging; OTR, ocular tilt reaction; VN, vestibular nuclei 

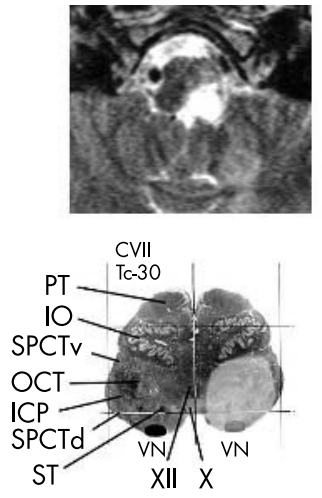

\# 1
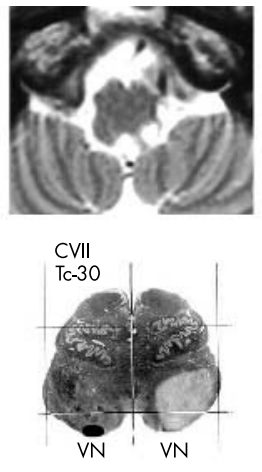

\#2
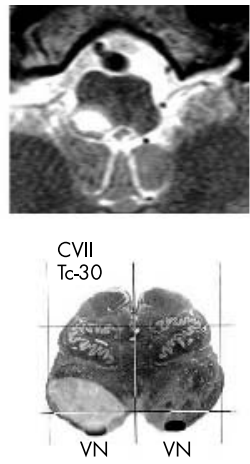

\#3
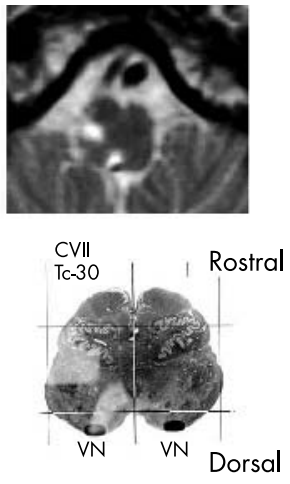

\#4

Figure 1 MRI. The T2 weighted axial magnetic resonance images of the medulla oblongata for patients \# 1 to \# 4 are shown in the first row. The extent of the lesion (white areas) is projected onto the appropriate anatomical slices (CVII, Tc-30) of the stereotaxic atlas of Schaltenbrand and Wahren. ${ }^{14}$ The location of the vestibular nuclei (VN) is marked as a black area. The lesion is a dorsal medullary infarction that involves the ipsilesional $\mathrm{VN}$ in all four patients. Important landmarks are indicated in the anatomical reconstruction of patient \#1 on the intact side. ICP, inferior cerebellar peduncle; IO, inferior olive; OCT, olivocerebellar tract; PT, pyramidal tract; SPCTd, dorsal spinocerebellar tract; SPCTv, ventral spinocerebellar tract; ST, nucleus of the solitary tract; X, dorsal motor vagal nucleus; XII, hypoglossal nucleus.

\section{Patient \#3}

A 73 year old hypertensive man presented with right lateral medullary syndrome. He had mild dysarthria and dysphagia, sensory loss of pain and temperature on the right side of his face, left trunk, and limbs, and hemiataxia of the right side. There was lateropulsion to the right. Oculomotor examination revealed nystagmus with a counterclockwise (contralesionally beating, leftward) torsional and a leftward horizontal component (fast phases) that increased in the dark. There was an upward vertical nystagmus component in both eyes. The nystagmus was conjugate and the amplitudes equal in both eyes. The patient had rightward lateropulsion of saccades (hypermetria), saccadic smooth pursuit eye movements in both directions, skew deviation with hypotropia of the right eye, and a head tilt to the right. MRI revealed a right dorsolateral medullary infarction (fig 1). Eye movement recordings were performed on the third day after symptom onset.

\section{Patient \#4}

A 63 year old man presented 2 days after onset of acute unsteadiness and vertigo with right dorsolateral medullary syndrome. He had right Horner's syndrome, sensory loss of pain and temperature on the left trunk and limbs, and hemiataxia of the right side. He also had a falling tendency to the right. Oculomotor examination demonstrated conjugate nystagmus with a counterclockwise (contralesionally beating, leftward) torsional, a rightward horizontal, and upward fast phase components in the light, which was more pronounced in the dark. The nystagmus was conjugate. There was also horizontal gaze evoked nystagmus in both horizontal directions. Saccades to the right were hypermetric. Skew deviation with hypotropia of the right eye was found. MRI revealed a right incomplete dorsolateral medullary infarction, with two parts, that is, a more dorsal and a more lateral portion (fig 1). Eye movement recordings were performed 5 days after symptom onset.

\section{MRI}

T1, T2, diffusion, and FLAIR weighted MRI including high resolution axial $3 \mathrm{~mm}$ slices of the brainstem were acquired (1.5 Tesla Siemens Magnetom Symphony, Germany). Reconstruction of the lesions was performed using axial and sagittal slices as described ${ }^{14}$ on the basis of the stereotaxic atlases of Schaltenbrand and Wahren ${ }^{13}$ and
Olszewski and Baxter. ${ }^{15}$ Individual axial slices were normalised and superimposed on the appropriate level of the anatomy atlas.

\section{Tonic ocular torsion}

Fundus photography was performed during mydriasis for each eye separately to measure tonic ocular torsion. Roll deviation of the fovea-macula intersection was measured in 10 age matched controls. More than $9.7^{\circ}$ or less than $1.4^{\circ}$ excyclorotation with respect to the horizontal meridian was considered as pathological.

\section{Search coil recording}

After the patients had given their informed consent, binocular, 3-D search coil recordings (Remmel, Ashland, MA, USA, frame size $180 \mathrm{~cm}$ cube; combination annulus, Skalar, Delft, the Netherlands) were performed. The head was stabilised comfortably in an upright position by a firm head rest and chin support. In vitro calibration of the coils and fixation at gaze straight ahead (laser target $145 \mathrm{~cm}$ distance from the eyes) was used to calibrate each individual eye. ${ }^{16}{ }^{17}$ The coil signals were digitised ( 16 bit AD, NI PCI $6071 \mathrm{E}, 600 \mathrm{~Hz}$ ) and filtered by an impulsive response filter at $100 \mathrm{~Hz}$ ( $3 \mathrm{~dB}$ level). Eye position was calculated in quaternions with eye velocity measured as angular not as derivative velocity. ${ }^{17}$ Clockwise (rightward) torsion (movement direction from the upper pole of the eye), downward, and leftward movements from the subject's view were defined as positive. The difference in vertical eye position between right and left eyes in gaze straight ahead while fixating with one eye was used to quantify the amount of skew deviation. Nystagmus was analysed in the light while the subject fixated at gaze straight ahead and in the dark.

To analyse the effect of the neural integrator on the nystagmus a single exponential function was fitted to the horizontal $(\mathrm{z})$, vertical $(\mathrm{y})$, and torsional $(\mathrm{x})$ eye positions of the slow phases using a least square minimisation procedure $\left(E(t)=O f f+E p * e^{\left(1 / T c^{*} t\right)} ; E\right.$, eye position; Off, positional offset; Ep, post saccadic eye position; Tc, time constant; $t$, time).$^{18}$ Each fit with a linear correlation coefficient over 0.95 was controlled manually and only those which aligned well with the position data were accepted. Negative Tc show an exponential decrease, positive Tc an increase of the slow phase of nystagmus. 
To analyse the vestibular contribution to the nystagmus, the median 3-D velocity of each slow phase in the light and dark was calculated. All slow phases with additional blinks were excluded. The analysis was performed with the fixating eye provided that the horizontal and vertical eye positions deviated less than $5^{\circ}$ from gaze straight ahead. The median slow phase velocity of each direction component was normalised by dividing the eye velocity components $(\mathrm{z}$,

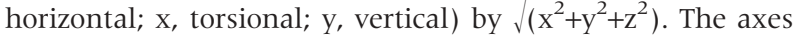
were compared to the axes of the semicircular canal stimulation using anatomical data. ${ }^{11}$ Based on Ewald's first law $^{19}$ (stimulation of a particular semicircular canal evokes eye movements in the plane of this semicircular canal, but in the opposite direction), we calculated the expected eye movement axis for individual semicircular canal stimulation. The deviation angle in 3-D of the slow phase eye rotation axis from the canal axes was calculated.

\section{RESULTS}

Three dimensional, binocular eye movements were examined in four patients who had acute dorsolateral medullary infarction and spontaneous jerk nystagmus. All patients had a lesion of the dorsolateral medulla which had been assessed on MRI (3-8 days after symptom onset), two on the right and two on the left side (fig l). There were no other lesions in the brainstem. Using the atlas of Schaltenbrand and Warren, ${ }^{13}$ reconstruction of the lesions showed that the vestibular nuclei were involved in all patients (fig 1); however, the lesion only partly involved the vestibular nuclei in two patients (\#3 and \#4). The medial vestibular nuclei involved in patients \#l-4 were identified by using the atlas of Olszewski and Baxter. ${ }^{15}$

The patients showed typical clinical signs of dorsolateral medullary syndrome. Three out of the four patients exhibited tonic ocular torsion in both eyes, which was always ipsilateral to the lesion. One patient (\#3) showed tonic ocular torsion only in one eye (table 1). There was always skew deviation of $1^{\circ}$ to $6^{\circ}$ with hypotropia ipsilateral and/or hypertropia contralateral to the lesion side (table 1 ).

All patients exhibited nystagmus with torsional, vertical, and horizontal components in gaze straight ahead (fig 2), which were on clinical observation more pronounced under Frenzel's glasses than in the light. The contralesional eye showed a larger torsional and the ipsilateral eye a larger vertical component in patients $\# 1$ and \#2. The torsional
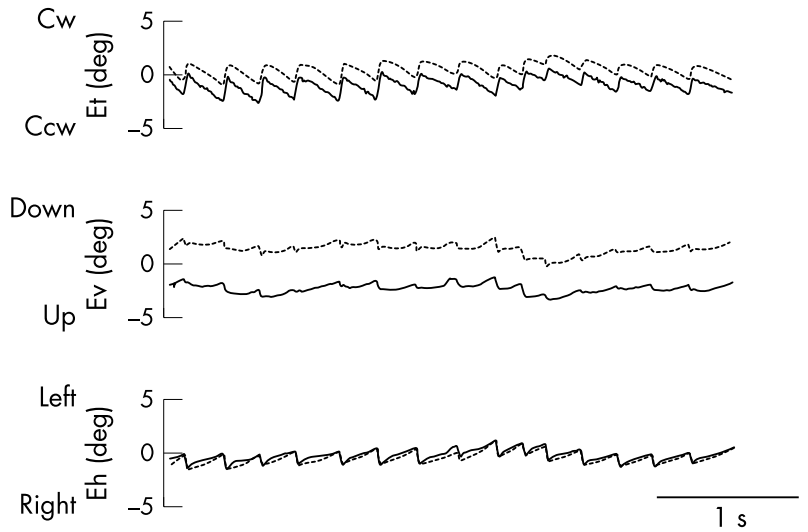

Figure 2 Nystagmus. Binocular search coil recordings of nystagmus in the light for patient \#1 are shown while he fixated at gaze straight ahead. Horizontal (Eh), vertical (Ev), and torsional (Et) eye positions are plotted for the right (solid line) and left (dotted line) eyes separately. Note the vertical skew deviation (second panel). Cw, clockwise (rightward) torsional; Ccw, counterclockwise (leftward) torsional.

component of the quick phases always beat to the contralesional side, clockwise (rightward) torsional for left sided lesions (patients \#1 and \#2) and counterclockwise (leftward) torsional for right sided lesions (patients \#3 and \#4). The vertical eye movement component was always upward in both eyes, the horizontal component contralesional in three of four subjects, and ipsilesional in one subject. The absolute average (SD) slow phase velocity of nystagmus in all patients in the dark was $7.2(4.4) \%$ (torsional $4.8(1.8) \%$; vertical 3.4 $(3.1) \%$; horizontal $3.4(3.7) \%$ ) and $6.2(4.5) \%$ (torsional 5.1 $(3.3) \%$; vertical $2.2(2.1) \%$; horizontal $2.6(2.6) \%)$ in the light (table 1).

Analysis of the exponential slow phases of nystagmus Most slow phases of the nystagmus were linear, but some, in all patients, also showed an exponential drift. Exponential increasing, decreasing, and even sigmoid shaped slow phases were found (fig 2). Time constants were calculated for selected single slow phases. Only slow phase fits which reached regression coefficients above 0.95 in all eye movement components were further analysed. In patients \# 1 and

Table 1 Static binocular misalignment and nystagmus slow phases

\begin{tabular}{|c|c|c|c|c|c|c|c|c|}
\hline \multirow{3}{*}{$\begin{array}{l}\text { Patient } \\
\text { Eye }\end{array}$} & \multirow{2}{*}{\multicolumn{2}{|c|}{$\begin{array}{l}\text { Left sided lesion } \\
\# \mathbf{1}\end{array}$}} & \multirow{2}{*}{\multicolumn{2}{|c|}{$\begin{array}{l}\text { Left sided lesion } \\
\# 2\end{array}$}} & \multirow{2}{*}{\multicolumn{2}{|c|}{$\begin{array}{l}\text { Right sided lesion } \\
\# 3\end{array}$}} & \multirow{2}{*}{\multicolumn{2}{|c|}{$\begin{array}{l}\text { Right sided lesion } \\
\# 4\end{array}$}} \\
\hline & & & & & & & & \\
\hline & $\overline{\mathrm{RE}}$ & LE & $\overline{\mathrm{RE}}$ & LE & $\overline{\mathrm{RE}}$ & LE & $\overline{\mathrm{RE}}$ & LE \\
\hline \multicolumn{9}{|c|}{ Static binocular alignment } \\
\hline Fundus $\left({ }^{\circ}\right)$ & $\operatorname{Cow} 5^{*}$ & $\mathrm{Ccw} 12^{*}$ & $\operatorname{Cow} 12^{*}$ & Cow $11^{*}$ & $\mathrm{Cw} 7$ & $\mathrm{Cw} 6^{*}$ & $\mathrm{Cw} 13^{*}$ & $\mathrm{Cw} 5^{*}$ \\
\hline Skew deviation & Fix & Hypotropia $4^{\circ}$ & Hypertropia $6^{\circ}$ & Fix & Hypotropia $1^{\circ}$ & Fix & Hypotropia $3^{\circ}$ & Fix \\
\hline \multicolumn{9}{|c|}{ Dark slow phase velocity of nystagmus } \\
\hline Xvel ( $\%$ s) & $-5.1(2.1)$ & $-7.7(3.6)$ & $-6.2(2.7)$ & $-5.9(2.8)$ & $4.0(1.3)$ & $3.6(0.6)$ & $3.3(1.5)$ & $2.3(1.3)$ \\
\hline Yvel $(\%)$ & $6.8(3.1)$ & $9.1(4.2)$ & $1.4(1.5)$ & $3.1(2.3)$ & $3.2(0.2)$ & $2.9(0.4)$ & $0.7(0.8)$ & $0.1(0.6)$ \\
\hline Zvel $(\%)$ & $8.8(1.7)$ & $9.6(2.3)$ & $1.5(1.9)$ & $1.2(1.6)$ & $-2.0(0.2)$ & $-3.6(1.3)$ & $-0.5(0.6)$ & $0.3(0.5)$ \\
\hline \multicolumn{9}{|c|}{ Light slow phase velocity of nystagmus } \\
\hline Xvel ( $\%$ s) & $-4.3(1.8)$ & $-5.1(2.1)$ & $-10.6(2.5)$ & $-9.8(4.3)$ & $3.0(1.4)$ & $2.4(1.3)$ & $3.0(1.4)$ & $2.4(1.3)$ \\
\hline Yvel $(\% \mathrm{~s})$ & $2.4(1.1)$ & $3.5(1.5)$ & $3.8(2.9)$ & 6.1 (3.4) & $0.7(0.6)$ & $0.2(0.6)$ & $0.7(0.6)$ & $0.2(0.6)$ \\
\hline Zvel ( $\%$ s) & $2.8(1.0)$ & $3.6(1.3)$ & $7.3(3.4)$ & $5.1(2.0)$ & $-0.5(0.4)$ & $-0.4(0.4)$ & $-0.5(0.4)$ & $0.4(0.4)$ \\
\hline
\end{tabular}

All values are given for each eye (LE, left eye; $R E$, right eye) and patient (\#1-4) separately. In the first row the tonic roll deviations of the eyes (fundus) are plotted. Pathological values of tonic roll deviation are marked by asterisks. The amount of skew deviation is shown and the fixating eye is indicated by "Fix". In the next rows the median slow phase velocity of the nystagmus in the dark and in the light is shown. Slow phase velocities: Xvel, torsional eye velocity; Yvel, vertical eye velocity; Zvel, horizontal eye velocity; Ccw, counter clockwise (leftward) torsional; $\mathrm{Cw}$, clockwise (rightward) torsional; Fix, fixating eye; positive, clockwise (rightward) torsion, left, downward. Values are mean (SD). 
\#2 there was more exponential increase in the vertical and torsional direction of the slow phases, in patients \#3 and \#4 more exponential decrease. However, in all patients some slow phases with either an exponential decrease or an exponential increase were found. Time constants were measured for those slow phases with a large exponential decay. On average the mean (SD) exponential decreasing time constants were: $-0.6(0.2) \mathrm{s}$ for horizontal, $-0.7(0.2) \mathrm{s}$ for vertical, and $-1.2(0.3) \mathrm{s}$ for torsional components; the mean (SD) increasing time constants were: $0.6(0.1) \mathrm{s}$ for horizontal, $0.3(0.1)$ s for vertical, and $0.4(0.1) \mathrm{s}$ for torsional components. In the majority of slow phases the fit of the single exponential function failed, because they were linear or had a more complex profile with an exponential and a linear component. Because of the linear slow phase, spontaneous nystagmus could not be explained by an unstable or impaired neural integrator alone.

\section{Analysis of the rotation axes of the slow phases}

The normalised slow phase velocity mainly reflects the late drift of the slow phase which is less contaminated by the exponential drifts. To test if the median slow phase velocity could be explained by semicircular canal imbalance, ${ }^{20}$ we compared the slow phase axes with the eye movement axes according to the stimulation of the individual semicircular canals. ${ }^{11}$

One example of the median slow phase axes is shown for patient \# $\mathrm{l}$ in the light (fig $3 \mathrm{~A}-\mathrm{C}$ ) and in the dark (fig 3D-F). For comparison, the expected eye movement axes of different semicircular canal stimulations are plotted. The slow phase axes for the right (filled circles) and left eyes (open squares) aligned between the right posterior ( $\mathrm{PC} \mathrm{r}$; thick solid line) and the right horizontal semicircular canals ( $\mathrm{HC} \mathrm{r}$; thick solid line). There was only a small but not significant difference between the axes in the light (fig 3A-C) and dark (fig 3D-F) or between both eyes. This patient $(\# 2)$ had a left sided lesion of the dorsolateral medulla (fig 1). Accordingly, the axes might reflect contralesional stimulation of the posterior (PC) and horizontal semicircular canals (HC) or an ipsilesional deficit of the anterior semicircular canal (AC) and HC.

The average traces of slow phase velocity for all four subjects (fig 4) showed a similar pattern. In fig 4 the nystagmus axes of all patients are normalised (see Methods) and presented as if the lesion was generally on the left side. All axes were aligned between the PC and HC of the contralesional side.

To test if the 3-D axes orientation of the slow phase is related to the semicircular canals, we calculated the 3-D deviation (in degrees) of the nystagmus slow phase axes from the expected axes as obtained by individual semicircular canal stimulation (fig 5). ${ }^{11}$ Large deviations were found in all subjects if the slow phase axes were compared to the expected axes of single contralesional (fig 5A-C) or ipsilesional semicircular canal stimulation (not shown). However, least deviation was found from a plane expected by simultaneous stimulation of the contralesional posterior and horizontal semicircular canals (fig 5D). The mean (SD) deviation from this combined canal plane was on average $14.7(5.0)^{\circ}$ (right eye: $15.2(5.0)^{\circ}$; left eye: $\left.14.3(5.5)^{9}\right)$ in the light and $20.3(8.5)^{\circ}$ (right eye: $21.2(8.1)^{\circ}$; left eye: 19.5 (10.0) 9 in the dark. This alignment was specific for the lesion side: left sided lesions showed nystagmus 3-D axes which closely aligned with those presumably elicited by the right sided PC and HC stimulation; right sided lesions showed nystagmus axes which aligned with those elicited by the left sided PC and HC. In summary, all patients exhibited a specific binocular nystagmus pattern: right and left sided lesions had an opposite axes pattern. This could be largely explained by stimulation of the PC and HC of the contralesional side, or by a lesion of the ipsilesional AC and HC.

\section{DISCUSSION}

This is the first time that the rotation axes of nystagmus have been analysed binocularly in acute dorsolateral medullary

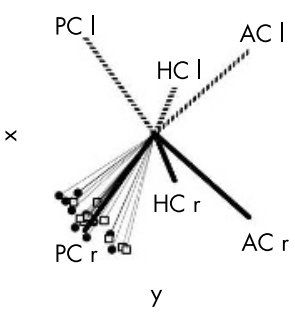

D

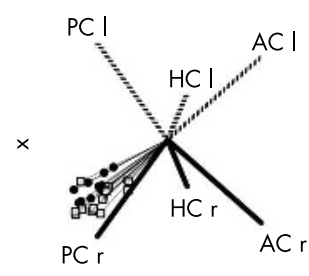

y

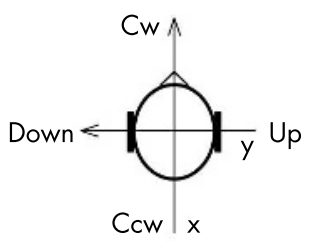

B

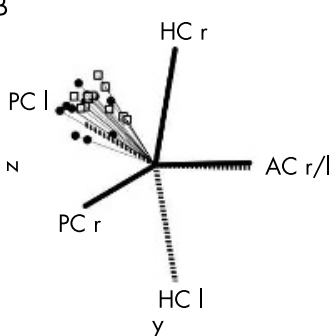

E
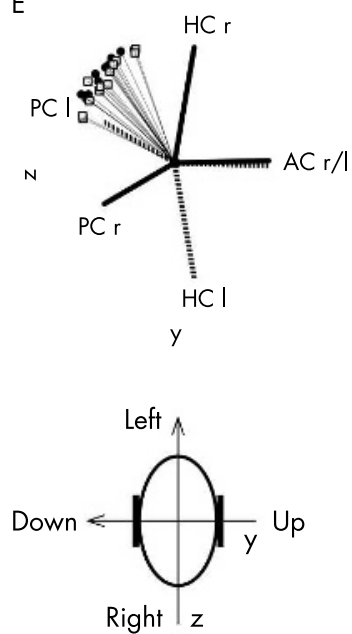

C

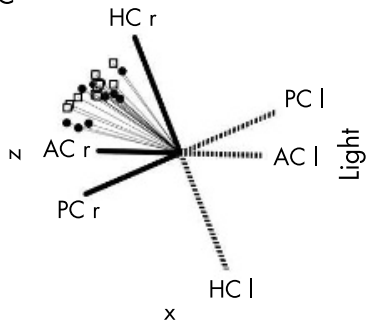

F
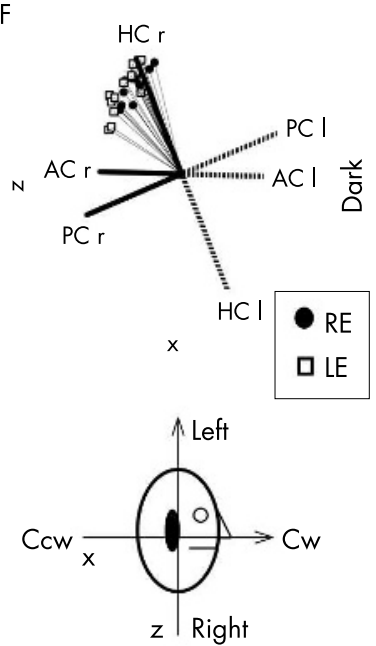

Figure 3 Rotation axes of spontaneous nystagmus (patient \#1). The median slow phases of spontaneous nystagmus are shown normalised (see Methods) for patient $\# 1$ in the light $(A-C)$ and the dark (D-F). The right eye (closed circles) and the left eye (open squares) are presented separately for each slow phase in three projections (schematic below). For comparison the expected rotation axes for semicircular canal stimulation of the right ( $r$; solid lines) or left (I; dashed lines) side are included (AC, anterior semicircular canal; $\mathrm{HC}$, horizontal semicircular canal; PC, posterior semicircular canal; LE, left eye; $R E$, right eye; l, left; $r$, right; $x$, torsional coordinate axis; $y$, vertical coordinate axis; $z$, horizontal coordinate axis). Slow phases are located in between the rotation axes, as obtained from anatomical data, ${ }^{1}$ of the contralesional posterior and horizontal semicircular canals. 


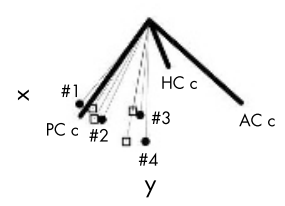

D
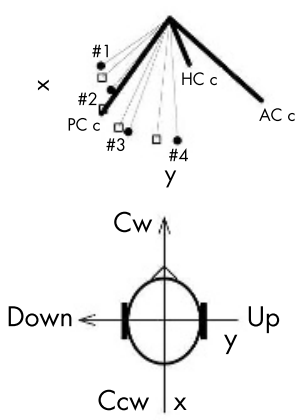

B

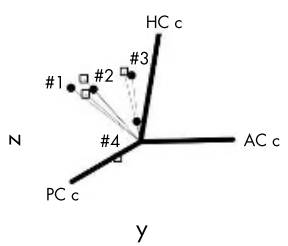

E

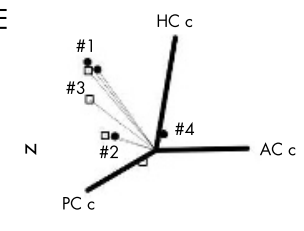

y

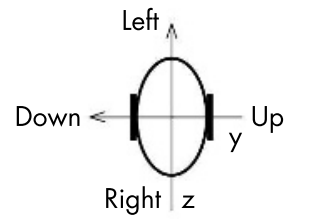

C

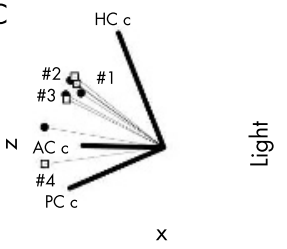

F
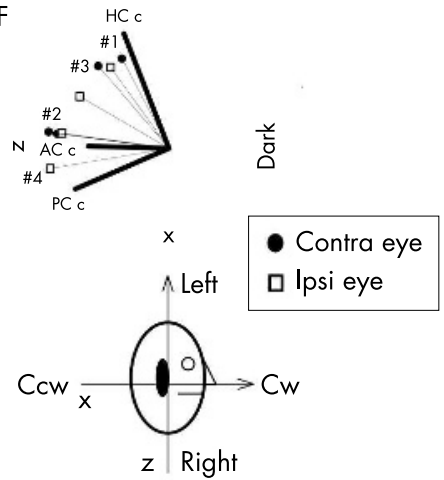

Figure 4 Rotation axes of spontaneous nystagmus (all patients). The slow phase axes of the spontaneous nystagmus of all subjects (\#1-\#4) are presented as if all the lesions were located on the left side. The mean normalised rotation axes of the slow phases of the contralesional eye (closed circles) and the ipsilesional eye (open squares) are shown in three different projections $(A-C)$. A schematic of the appropriate projection is indicated below $(x$, torsional coordinate axis; $y$, vertical coordinate axis; $z$, horizontal coordinate axis). For comparison, the predicted movement axes of contralesional (right) semicircular canal stimulation are plotted (solid line; AC, anterior semicircular canal; $\mathrm{HC}$, horizontal semicircular canal; PC, posterior semicircular canal; $C$, contralesional). infarction to determine whether symptoms in dorsolateral medullary infarction could be explained by imbalance of the semicircular canals in addition to the imbalance of the central otolith pathways. ${ }^{125}$ The clinical and oculomotor findings in all of the patients were compatible with a dorsolateral medullary infarction as described elsewhere. ${ }^{2}$ Accordingly, all patients had saccadic lateropulsion, skew deviation with an ipsilesional hypotropic eye, and in three out of four patients, tonic ocular torsion. All patients had ipsilesional lateropulsion of the body. MRI slices showed a unilateral dorsolateral medullary infarction in all patients. According to the anatomical reconstruction on MRI slices, the medial vestibular nuclei were affected on one side in all patients and possibly the vestibular commissural pathways in all patients.

\section{Neural integrator and nystagmus}

As described earlier, ${ }^{6}$ we mainly found linear, but also some exponentially decreasing or increasing, slow phases of nystagmus at gaze straight ahead. Exponentially decreasing slow phases are a sign of neural integrator failure: the eye velocity signal is no longer integrated into an appropriate eye position signal. In contrast, exponentially increasing slow phases are a sign of an unstable neural integrator..$^{21} 22$ The neural integrator for the horizontal eye movements is mainly located in the nucleus prepositus hypoglossi and in the vestibular nuclei, ${ }^{21}$ and for the vertical and the torsional eye movements in the interstitial nucleus of $\mathrm{Cajal}^{18}$ and the vestibular nuclei. ${ }^{23}$ Lesions of the medial vestibular nuclei and the nucleus prepositus hypoglossi usually cause contralesional nystagmus at gaze straight ahead and could lead to horizontal integrator failure, with time constants of the slow phases as slow as $200 \mathrm{~ms}^{21}{ }^{24}$ Integrator failure, however, could only partly explain the nystagmus in our patients because a considerable portion of the slow phases were linear. Thus, an additional vestibular origin of the nystagmus is likely.

\section{Vestibular nystagmus}

Some evidence to support the nystagmus in our patients being of vestibular origin comes from the finding that it is enhanced in the dark. ${ }^{22}$ The 3-D components of nystagmus in our patients had the same direction characteristics as described previously. ${ }^{6}$ These authors suspected a vestibular origin. The torsional quick phase was always opposite to the lesion side and the vertical quick phases had an upward direction. The horizontal component in three of our four patients was contralesional and in one patient (\#4) ipsilesional but of very small horizontal slow phase velocity. The nystagmus direction in our patients, that is, contralesional, is in accordance with experimental lesion of the vestibular nuclei. $^{25}$

To determine the contribution of the semicircular canals to the nystagmus in our patients we compared the rotation axes of nystagmus slow phases with the expected stimulation axes as derived from anatomical data of the semicircular canals. ${ }^{11}$ Analysis of the binocular rotation axes of the eyes with detailed 3-D recordings in patients with vestibular nuclei lesions has not yet been performed.

Stimulation of the semicircular canal afferents always elicits an eye movement in the plane of the canal, but in the opposite direction (Ewald's first law). ${ }^{19}$ The nystagmus slow phase axes in our patients did not align with those of single semicircular canal stimulation. However, combined stimulation of the posterior and the horizontal semicircular canals based on anatomical data ${ }^{11}$ closely aligns with the slow phase axes obtained in our patients. There is some evidence that semicircular canals may contribute to spontaneous nystagmus in dorsolateral medullary syndrome. The spontaneous nystagmus might be caused by central imbalance of the semicircular canals, either by a lesion of the ipsilesional horizontal and anterior semicircular canals or by stimulation of the contralesional horizontal and posterior semicircular canals.

The small deviation of the slow phase axes from the canal plane could be caused by (i) tonic ocular torsion and skew deviation, (ii) the contribution of the otoliths (utriculus and sacculus), or (iii) the anterior semicircular canal (AC). The tonic ocular torsion was quite small (table 1). This small tonic ocular torsion cannot account for deviation of the slow phase axes large enough to cause the measured axes pattern. ${ }^{12}$

It is uncertain whether central imbalance of otolith signals causes nystagmus or if the otolith signals only influence an existing imbalance of the semicircular canals. ${ }^{26}$ While the nystagmus in our patients is probably not caused by utricular imbalance alone, ${ }^{12}$ there might be some utricular or saccular contribution accounting for the residual axes deviation. In the vestibular nuclei there is some convergence of the 

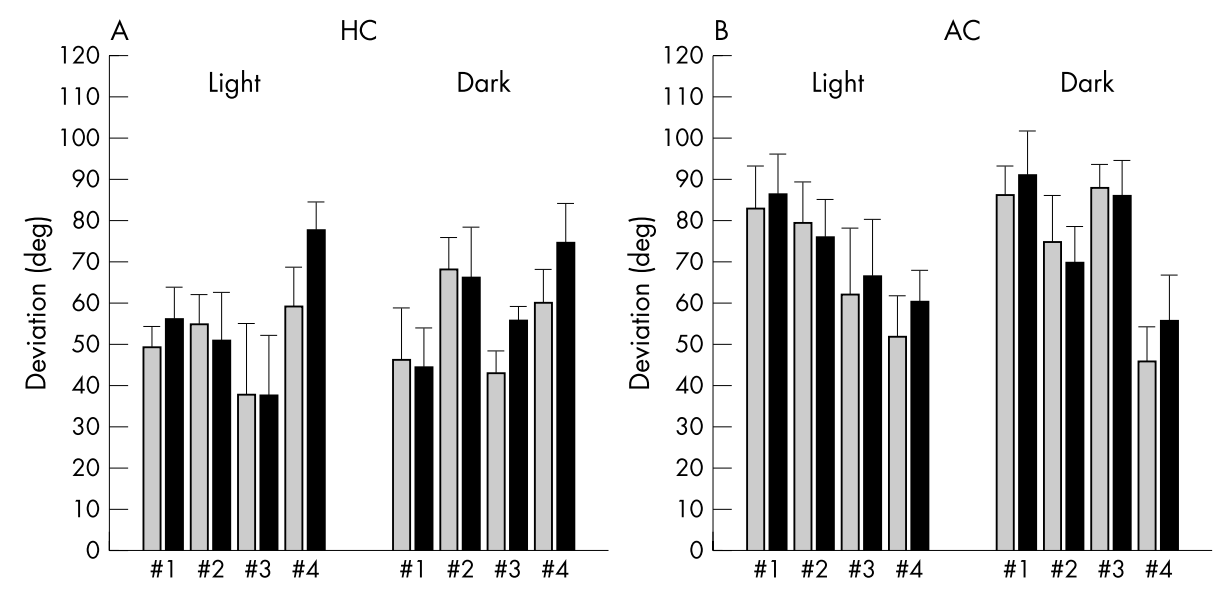

Figure 5 Deviation of the slow phase axis of nystagmus from semicircular canals. The 3-D angle between the expected axes of the semicircular canals and the mean slow phase axis in the light is plotted for each patient $(\# 1-4)$ and for the ispsilesional (Ipsi; hatched columns) and contralesional eyes (Contra; filled columns) separately. The deviations of the slow phase axes from the predicted axes from the individual semicircular canal (AC, anterior semicircular canal; $\mathrm{HC}$, horizontal semicircular canal; PC, posterior semicircular canal) stimulation axes are shown in $(A-C)$, the combination of the posterior and the horizontal semicircular canal stimulation in (D). Least deviation is shown in (D) for all patients.
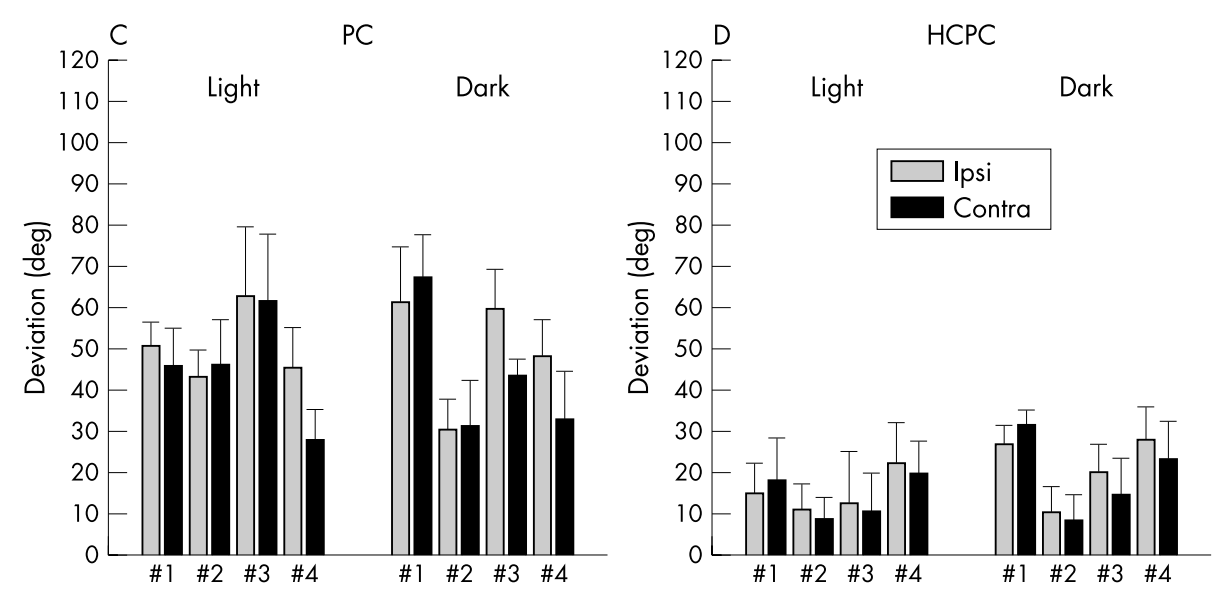

utricular and the posterior ${ }^{27}$ and, to some extent, the horizontal ${ }^{28}$ semicircular canal afferents which may contribute to our nystagmus. There might be some additional contribution of the anterior semicircular canal to the axis deviation.

Our data are in accordance with previous reports obtained from 2-D electro-oculography and clinical assessments which proposed that torsional nystagmus in brainstem lesions might be caused by central vertical semicircular imbalance and not by imbalance of the otoliths. ${ }^{10}$ This finding is in line with the small contribution of the otoliths to physiological torsional nystagmus. ${ }^{29}$ More specifically according to our analysis, the major contribution to the nystagmus slow phase axes in straight ahead gaze may be caused by the combined imbalance of the horizontal and vertical semicircular canals, for example, indicating a lesion of the ipsilesional anterior and horizontal semicircular canals. Whether the nystagmus of our patients is caused by an immediate lesion of the vestibular nuclei and their pathways, or by lesion of the vestibulo-cerebellar projections remains to be determined.

\section{CONCLUSION}

We provide evidence that there is central semicircular canal imbalance in addition to otolith imbalance in dorsolateral medullary infarctions. ${ }^{2}$ The direction of the spontaneous nystagmus in the first days after infarction is consistent with combined stimulation of the horizontal and posterior semicircular canals on the contralesional side.

\section{ACKNOWLEDGEMENTS}

The authors thank Mrs J Benson for carefully reading of the manuscript.

\section{Authors' affiliations}

H Rambold, C Helmchen, Department of Neurology, University of Luebeck, Luebeck, Germany

Supported by Deutsche Forschungsgemeinschaft (DFG)

Competing interests: none declared

\section{REFERENCES}

1 Waespe W, Wichmann W. Oculomotor disturbances during visualvestibular interaction in Wallenberg's lateral medullary syndrome. Brain 1990;113(Pt 3):821-46.

2 Dieterich M, Brandt T. Wallenberg's syndrome: lateropulsion, cyclorotation, and subjective visual vertical in thirty-six patients. Ann Neurol 1992;31:399-408.

3 Brandt T, Dieterich M. Pathological eye-head coordination in roll: tonic ocular tilt reaction in mesencephalic and medullary lesions. Brain 1987; 110(P+ 3):649-66.

4 Gresty MA, Bronstein AM, Brandt T, et al. Neurology of otolith function. Peripheral and central disorders. Brain 1992;115(Pt 3):647-73.

5 Brandt TH, Dieterich M. Different types of skew deviation. J Neurol Neurosurg Psychiatry 1991;54:549-50.

6 Morrow MJ, Sharpe JA. Torsional nystagmus in the lateral medullary syndrome. Ann Neurol 1988;24:390-8.

7 Estanol B, Lopez-Rios G. Neuro-otology of the lateral medullary infarct syndrome. Arch Neurol 1982;39:176-9.

8 Baloh RW, Yee RD, Honrubia V. Eye movements in patients with Wallenberg's syndrome. Ann N'Y Acad Sci 1981;374:600-13.

9 Kommerell G, Hoyt WF. Lateropulsion of saccadic eye movements. Electrooculographic studies in a patient with Wallenberg's syndrome. Arch Neurol 1973;28:313-8.

10 Lopez L, Bronstein AM, Gresty MA, et al. Torsional nystagmus. A neuro-otological and MRI study of thirty-five cases. Brain 1992; 1 15(Pt 4): 1 107-24.

11 Blanks RH, Curthoys IS, Markham CH. Planar relationships of the semicircular canals in man. Acta Otolaryngol 1975;80:185-96.

12 Rambold H, Helmchen C. 3-D aspects of spontaneous nystagmus in dorsolateral medullary infarction. Ann N Y Acad Sci 2003;1004:497-9.

13 Schaltenbrand G, Wahren W. Atlas of the stereotaxy of human brain. Stuttgart: Thieme, 1977. 
14 Fitzek S, Baumgartner U, Fitzek $C$, et al. Mechanisms and predictors of chronic facial pain in lateral medullary infarction. Ann Neurol 2001;49:493-500.

15 Olszewski J, Baxter B. Cytoarchitecture of the human brain stem. Basel: Karger, 1982.

16 Rambold H, Kompf D, Helmchen C. Convergence retraction nystagmus: a disorder of vergence? Ann Neurol 2001;50:677-81.

17 Tweed D, Vilis T. Implications of rotational kinematics for the oculomotor system in three dimensions. J Neurophysiol 1987;58:832-49.

18 Helmchen C, Rambold H, Fuhry L, et al. Deficits in vertical and torsional eye movements after uni- and bilateral muscimol inactivation of the interstitial nucleus of Cajal of the alert monkey. Exp Brain Res 1998;1 19:436-52.

19 Ewald JR. Physiologische Untersuchungen veber das Endorgan des Nervus octavus. Wiesbaden: Bergmann, 1892.

20 Rambold H, Helmchen C, Buttner U. Vestibular influence on the binocular control of vertical-torsional nystagmus after lesions in the interstitial nucleus of Cajal. Neuroreport 2000;11:779-84.

21 Cannon SC, Robinson DA. Loss of the neural integrator of the oculomotor system from brain stem lesions in monkey. J Neurophysiol 1987; 57:1383-409.
22 Leigh RJ, Zee DS. The neurology of eye movements. New York: Oxford University Press, 1999

23 Fukushima K, Kaneko CR. Vestibular integrators in the oculomotor system. Neurosci Res 1995;22:249-58.

24 Mettens P, Godaux E, Cheron G, et al. Effect of muscimol microinjections into the prepositus hypoglossi and the medial vestibular nuclei on cat eye movements. J Neurophysiol 1994;72:785-802.

25 Uemura T, Cohen B. Effects of vestibular nuclei lesions on vestibulo-ocular reflexes and posture in monkeys. Acta Otolaryngol Suppl 1973;315:1-71.

26 Gresty M, Barratt $H$, Rudge $P$, et al. Analysis of downbeat nystagmus. Otolithic vs semicircular canal influences. Arch Neurol 1986;43:52-5.

27 Zakir M, Kushiro K, Ogawa Y, et al. Convergence patterns of the posterior semicircular canal and utricular inputs in single vestibular neurons in cats. Exp Brain Res 2000;132:139-48.

28 Zhang $\mathrm{X}$, Zakir $\mathrm{M}, \mathrm{Meng} \mathrm{H}$, et al. Convergence of the horizontal semicircular canal and otolith afferents on cat single vestibular neurons. Exp Brain Res $2001 ; 140: 1-11$.

29 Jauregui-Renaud K, Faldon M, Clarke A, et al. Skew deviation of the eyes in normal human subjects induced by semicircular canal stimulation. Neurosci Lett 1996;205:135-7.

\section{$\mathrm{ECHO}$}

\section{Training of care givers after stroke reduces costs}

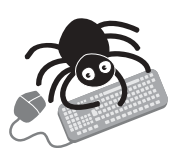

Please visit the Journal of Neurology, Neurosurgery, and Psychiatry website [jnnp.com] for a link to the full text of this article. mprovements in stroke rehabilitation have increased the number of disabled patients who live at home with the help of untrained care givers. Trying to help care givers by providing information packages, family support workers, or specialist nurses has provided only modest benefits for patients and carers. Now researchers in London, UK have shown that caregiver training is beneficial and reduces service costs.

The care givers of 300 stroke patients on a stroke rehabilitation unit were randomised, before the patient went home, to training in basic nursing, moving, handling, and assistance with activities of daily living and communication (three to five 30-45 minute sessions on the unit and one follow up session at home) or no training. All received conventional advice, information, and involvement. Caregivers who received training had better mental health and quality of life at three and 12 months and the patients' quality of life also improved.

The economic evaluation included health services, other formal care agencies, and informal carers and cost per patient was calculated from unit costs and resource volumes. Health and social costs over one year at 2001-2002 prices were $£ 10544$ (caregiver training) $v$ $£ 14587$ (no training), a saving of $£ 4043$ (US $\$ 7249, € 6072$ ). With inclusion of informal care costs the total saving was $£ 4091$. Most of the saving was due to shorter initial hospital stay in the caregiver training group rather than cost reductions in care whilst at home. The costs of informal care were similar in the two groups so there was no shifting of costs from statutory services to care givers. No change in caregivers' quality adjusted life years was detected using the EuroQol five-dimensional questionnaire (EQ-5D).

Providing training for caregivers of stroke patients improves their quality of life, as well as that of the patients, and reduces service costs, largely by expediting discharge from hospital. $\Delta$ Patel A, et al. BMJ 2004;328:1102-1104. 